\title{
Endoscopic dilatation as a new technique in managing pediatric duodenal hematoma
}

\author{
Fahad M. Alharbi, MD, Zaki A. Abo Amer, MD, Khalel H. Hamamesh, MD, Sarah N. Algubaisi, MD.
}

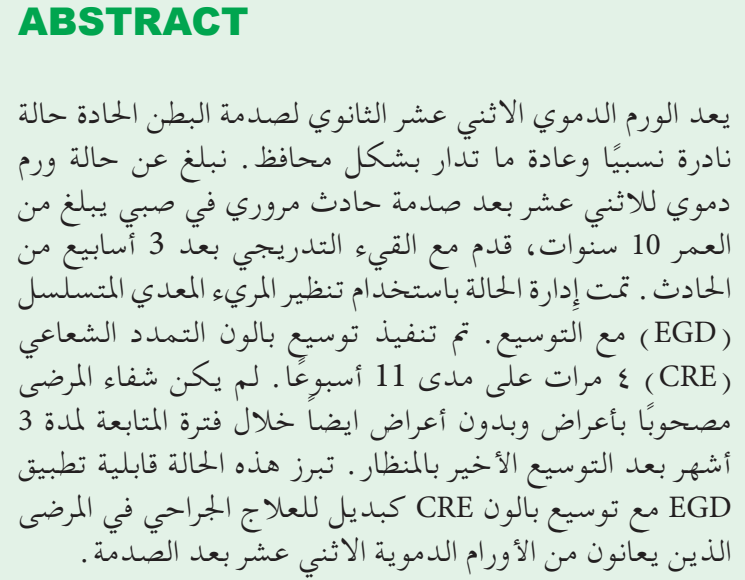

A duodenal hematoma secondary to blunt -abdominal trauma is a relatively rare condition and is usually managed conservatively. We report a case of a post-traumatic duodenal hematoma after a road traffic accident in a 10 -year-old boy, who presented with progressive vomiting 3 weeks after the accident. The case was managed using serial esophagogastroduodenoscopy (EGD) with dilatation. Controlled radial expansion (CRE) balloon dilatation was performed 4 times over a period of 11 weeks. The patient recovered uneventfully and remained asymptomatic at the 3-month follow-up after the last endoscopic dilatation. This case highlights the applicability of EGD with CRE balloon dilatation as an alternative to surgical treatment in patients with symptomatic post-traumatic duodenal hematomas.

Keywords: duodenum, hematoma, endoscopy, dilatation

Saudi Med J 2020; Vol. 41 (8): 874-877 doi: $10.15537 /$ smj.2020.8.25128

From the Department of Pediatrics (Alharbi, Algubaisi), Prince Sultan Military Medical City, Riyadh; from the Department of Pediatrics (Abo Amer); and from the Department of Surgery (Hamamesh), Al-Kharj Military Hospital, Al Kharj, Kingdom of Saudi Arabia.

Received 5th March 2020. Accepted 29th April 2020.
Address correspondence and reprint request to: Dr. Fahad M. Alharbi, Department of Pediatrics, Prince Sultan Military Medical City, Riyadh, Kingdom of Saudi Arabia. E-mail: dr.fahadalharbi@outlook.com ORCID ID: orcid.org/0000-0003-1678-4623

Tntramural duodenal hematomas (DHs) are relatively rare in the pediatric population, observed in only $3-5 \%$ of cases of abdominal injury in children. ${ }^{1-3}$ Commonly, the duodenum is compressed between the colliding object and the vertebral column during the injury. ${ }^{3}$ The most widely recognized causes of trauma leading to $\mathrm{DH}$ in the pediatric population include road traffic accidents (RTAs) and abuse. ${ }^{3}$ Intramural duodenal hematomas usually present within a few days of the trauma, with conservative management being the first choice of treatment. In general, surgical management is reserved for patients with perforation, other injuries requiring surgical intervention, or with persistent symptoms such as feeding intolerance or vomiting that do not resolve within 3 weeks. ${ }^{4,5}$ We present a case of $\mathrm{DH}$ in a child, aiming to describe a novel treatment using endoscopic balloon dilatation. This treatment strategy is less invasive and may serve as an alternative to surgery.

Case Report. A healthy 10-year-old boy presented with a right forearm fracture after an RTA; he exhibited no signs of abdominal pain or vomiting. An abdominal computed tomography (CT) scan was performed at the time of admission. Although he had developed a DH, this was missed during CT screening (Figure 1).

Clinical findings. Laboratory evaluations indicated a hemoglobin level of $12.2 \mathrm{~g} / \mathrm{dl}$, an alanine transaminase (ALT) level of $259 \mathrm{U} / \mathrm{L}$, and an aspartate aminotransferase (AST) level of $412 \mathrm{U} / \mathrm{L}$; this was thought to be related to the RTA. Other laboratory evaluations indicated that the renal profile, amylase level, and coagulation profile were within the normal range. Hemoglobin level remained stable, while ALT and AST levels decreased during admission. He exhibited no further symptoms 


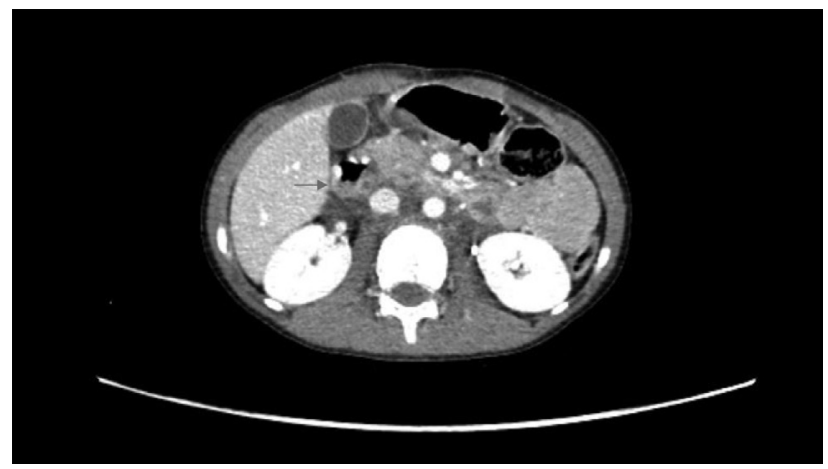

Figure 1 - First computed tomography scan of abdomen: showing dilated wall of duodenum.

and was discharged after 2 days with a cast on the right forearm; no other interventions were considered necessary.

Diagnostic assessment. Three days after discharge, the patient started to vomit. The frequency of vomiting increased with time and persisted for 27 days before medical advice was sought. The patient presented to our emergency department with persistent nonbilious vomiting, abdominal pain, inability to tolerate feeding, and weight loss of $2 \mathrm{~kg}$. He was considered to have an intestinal obstruction. A CT scan was performed, this time revealing a $\mathrm{DH}$ causing narrowing of the duodenal lumen (Figure 2). His hemoglobin level was maintained, and his ALT and AST levels, amylase levels, and coagulation profile were within the normal range. He was kept nil per os (NPO) for 5 days; however, his clinical symptoms did not improve.

$$
\text { Therapeutic intervention. }
$$

Pediatric esophagogastroduodenoscopy (EGD) was performed, revealing severe stenosis in the third portion of the duodenum; the pediatric gastroscope with an outer diameter of $8.9 \mathrm{~mm}$ could not pass through. A controlled radial expansion (CRE) balloon was dilated to 15 $\mathrm{mm}$ without complications (Figures 3-5). Omeprazole was initiated at a daily dose of $1 \mathrm{mg} / \mathrm{kg}$. The patient tolerated full oral intake and was discharged 2 days after the dilatation procedure with a follow-up EGD appointment in 2 weeks. During the EGD follow-up, a recurrence of the severe stenosis in the same area was detected, and CRE balloon dilatation to $18 \mathrm{~mm}$ was performed without complications. He underwent 2 more sessions of endoscopic CRE dilatation, and

Disclosure. Authors have no conflict of interests, and the work was not supported or funded by any drug company.

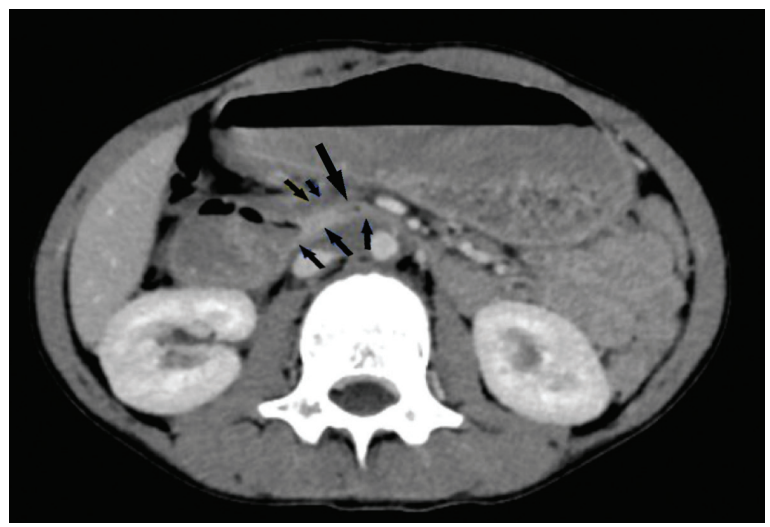

Figure 2 - Second computed tomography scan of abdomen showing dilated stomach and first and second part duodenum and stenosis in third part of duodenum (arrows).

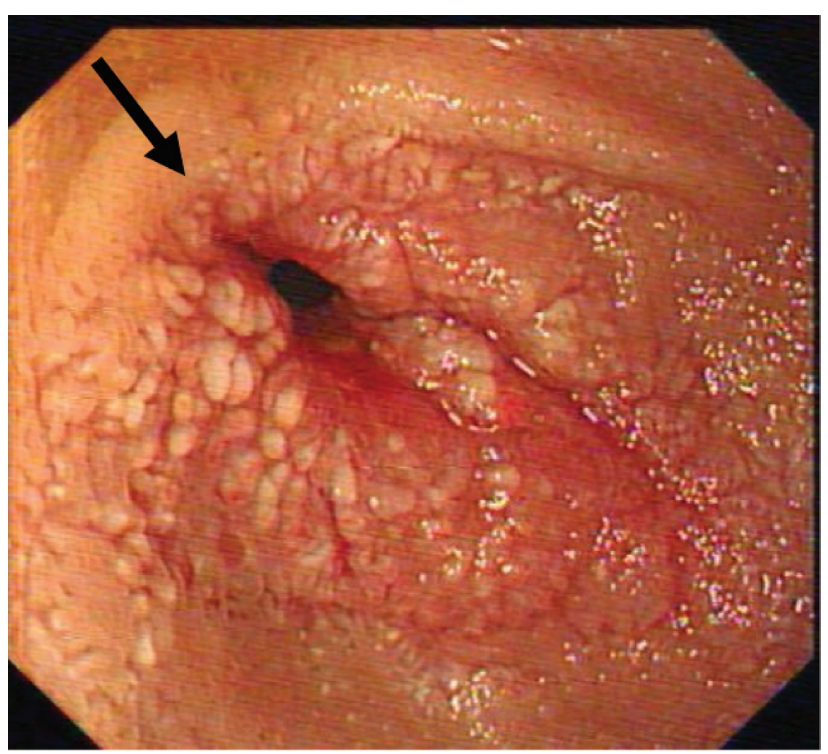

Figure 3 - First esophagogastroduodenoscopy pre-dilatation image showing the stenosis of the duodenal lumen (arrow).

a better outcome for the outer diameter of the third portion of the duodenum was achieved at the end of the fourth session.

Follow-up and outcomes. At the fifth EGD (performed 11 weeks after the first EGD), no stenosis was detected and no interventions were required (Figure 6). He remained asymptomatic throughout the 3-month follow-up period in our outpatient clinic and experienced improved weight gain.

Discussion. We were successfully able to manage the $\mathrm{DH}$ via $\mathrm{EGD}$ with endoscopic balloon dilatation. While a patient's history and physical assessment may 


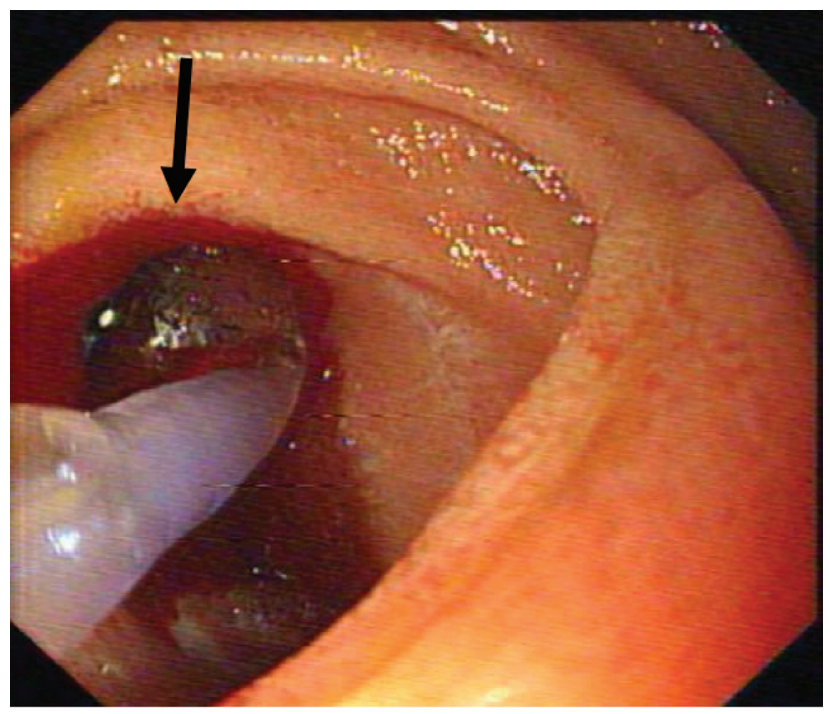

Figure 4 - First esophagogastroduodenoscopy showing controlled radial expansion balloon dilatation of the stricture of the duodenal lumen (arrow).

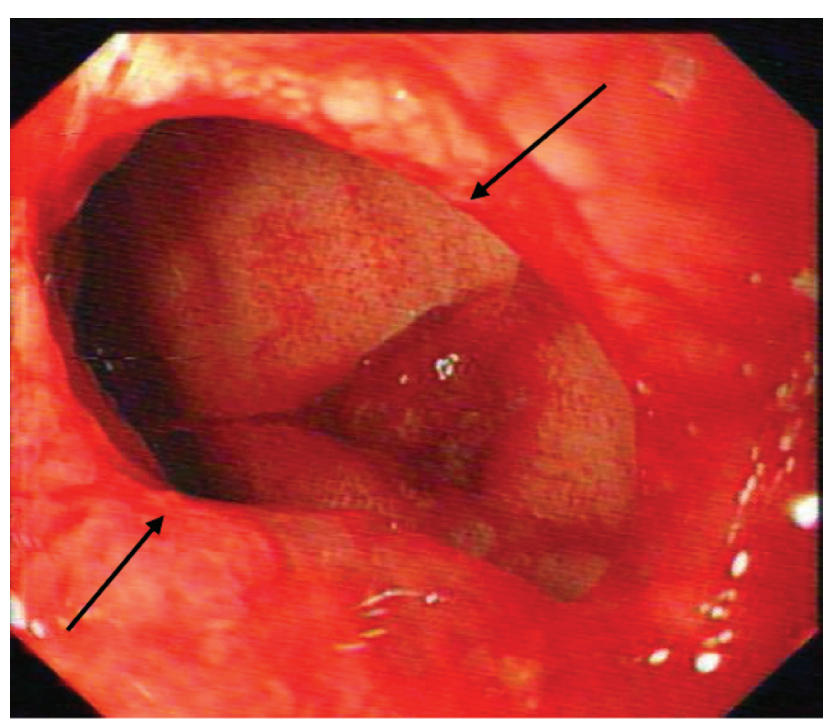

Figure 5 - First esophagogastroduodenoscopy post-balloon dilatation image showing improvement of the stricture of the duodenal lumen (arrows).

aid in detecting the cause of the duodenal obstruction, a radiological study is needed to confirm the diagnosis. ${ }^{6}$ Ultrasound may be used, which will show a thickened wall or an obstruction of the lumen. An upper gastrointestinal series may also aid in identifying the obstructed area; however, a diagnosis cannot be achieved using this method. ${ }^{6,7}$ Computed tomography scan remains the gold standard for establishing the diagnosis of $\mathrm{DH}$ as heterogeneous attenuation of

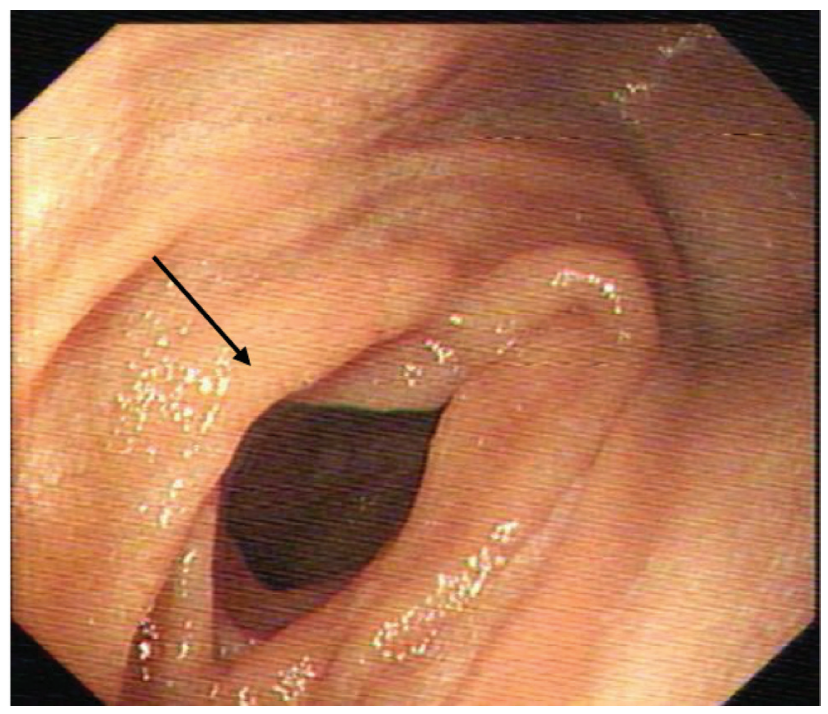

Figure 6 - Last esophagogastroduodenoscopy showing a complete resolution of the stricture and normal duodenal lumen (arrow).

the duodenal wall may be observed and will aid in ruling out perforation. ${ }^{6}$ Recurrent abdominal pain, vomiting, and feeding intolerance are noted in children who exhibit late presentation, as seen in our case. ${ }^{4}$ Intramural duodenal hematomas are usually treated conservatively by keeping the patient NPO, starting total parenteral nutrition (TPN), and performing nasogastric tube decompression; in most cases, the patient resumes a normal diet after an average of 2-3 weeks. ${ }^{4,5}$ However, surgical treatment is required in cases of bowel perforation or where symptoms persist after 2 to 3 weeks. ${ }^{2,3}$

Another management modality that may be used after the failure of conservative management is CRE balloon dilatation, although this procedure is not widely used, as noted upon review of the available literature. Samra et $\mathrm{al},{ }^{8}$ reported successful treatment of an intramural hematoma in an adult patient via the simple passage of the gastroscope; this passage aided in opening of the duodenal stricture, suggesting the possibility of endoscopic balloon dilation as another treatment method.

Our patient was a survivor of an RTA with a delayed presentation (21 days after the RTA) of intestinal obstruction. The obstruction was managed using serial endoscopic balloon dilatation over a period of 11 weeks. The patient was successfully discharged 1-2 days after each procedure, that is, with a decreased hospitalization duration, and the patient showed good oral intake. During the 3-month follow-up period after the last endoscopic dilatation, the patient remained 
asymptomatic with rapid weight gain. Controlled radial expansion balloon dilatation was performed successfully with no immediate or late complications. This novel modality may, therefore, be used to treat cases of $\mathrm{DH}$ instead of surgical management.

Study limitations. First, this specific approach to treatment has not been previously described and is thus not standardized. Second, further research is required to confirm the efficacy of this technique.

In conclusion, EGD with dilatation of a CRE balloon for management of a DH is feasible, safe, and effective and could help avoid surgical intervention. We believe that this approach could herald a change in the practice of DH management.

Acknowledgment. The authors gratefully acknowledge Dr. Bashar A. Ahmad, consultant pediatric radiologist, in Prince Sultan Military Medical City, for reviewing the CT scan images and we would like to thank (www.editage.com) for editing service.

\section{References}

1. Sahn B, Anupindi SA, Dadhania NJ, Kelsen JR, Nance ML, Mamula P. Duodenal hematoma following EGD: comparison with blunt abdominal trauma-induced duodenal hematoma. $J$ Pediatr Gastroenterol Nutr 2015; 60: 69-74.
2. Banieghbal B, Vermaak C, Beale P. Laparoscopic drainage of a post-traumatic intramural duodenal hematoma in a child. $J$ Laparoendosc Adv Surg Tech A 2008; 18: 469-472.

3. Moon SB, Jung SE, Cheon JE, Kim WS, Park KW. Very large traumatic duodenal hematoma: successful percutaneous drainage in a child. Eur J Pediatr Surg 2009; 19: 412-413.

4. Torba M, Gjata A, Buci S, Troci A, Subashi K. Delayed presentation of blunt duodenal injuries in children. Case report and review of literature. G Chir 2013; 34: 122-124.

5. Peterson ML, Abbas PI, Fallon SC, Naik-Mathuria BJ, Rodriguez JR. Management of traumatic duodenal hematomas in children. J Surg Res 2015; 199: 126-129.

6. Niehues SM, Denecke T, Bassir C, Hamm B, Haas M. Intramural duodenal hematoma: clinical course and imaging findings. Acta Radiol Open 2019; 8: 2058460119836256.

7. Homma Y, Mori K, Ohnishi Y, Fujioka K, Terada T, Sasaki A, et al. Ultrasound follow-up in a patient with intestinal obstruction due to post-traumatic intramural duodenal hematoma. J Med Ultrason (2001) 2016; 43: 431-434.

8. Samra M, Al-Mouradi T, Berkelhammer C. Gastric outlet obstruction due to intramural duodenal hematoma after endoscopic biopsy: Possible therapeutic role of endoscopic dilation. Case Rep Gastroenterol 2018; 12: 692-698. 